\title{
The asymptotic expansion of the regular discretization error of Itô integrals
}

\author{
Elisa Alòs* \\ Dpt. d'Economia i Empresa \\ Universitat Pompeu Fabra \\ and Barcelona GSE \\ c/Ramon Trias Fargas, 25-27 \\ 08005 Barcelona, Spain \\ elisa.alos@upf.edu \\ Masaaki Fukasawa \\ Graduate School of Engineering Science \\ Osaka University \\ 1-3 Machikaneyama, Toyonaka \\ 560-8531 Osaka, Japan \\ fukasawa@sigmath.es.osaka-u.ac.jp
}

February 22, 2018

\begin{abstract}
We study a Edgeworth-type refinement of the central limit theorem for the discretizacion error of Itô integrals. Towards this end, we introduce a new approach, based on the anticipating Itô formula. This alternative technique allows us to compute explicitly the terms of the corresponding expansion formula.
\end{abstract}

Keywords: Itô integral, discretization error, central limit theorems, Malliavin calculus.

AMS subject classification: 60F05, 60H05, 60H07.

\section{Introduction}

The Itô integral for semi-martingales is defined as a limit of a random sequence of Riemann-Stieltjes type sum. First it is defined as an $L^{2}$ limit and then, the

*Supported by the Spanish grant MTM2013-40782-P. 
limit is characterized also in terms of convergence in probability in a functional sense. A natural question, from both theoretical and practical viewpoints, is how close the limit and the Riemann-Stieltjes type sum are. The difference between them, which we call the discretization error, should converge to 0 as the time partition for the Riemann-Stieltjes sum becomes finer and finer. The question is how fast it is and how a renormalized error behaves.

The first answer to this question was given by Rootzén [9], where a central limit theorem for the regular discretization error, that is, the discretization error when the time partition is regular; $0,1 / n, 2 / n, \ldots$ was proven. The convergence rate is $\sqrt{n}$ and the limit distribution is mixed normal. The convergence holds stably and the theory of stable convergences for discretized processes to conditionally Gaussian processes was further developed by Jacod and Shiryaev [4]. The theory has remarkable applications to high-frequency data analysis; see e.g. Aït-Sahalia and Jacod [1] and Jacod and Protter [3]. The general theory also enables us to treat irregular and stochastic time partitions for the RiemannStieltjes sum; see Fukasawa [2]. In the context of mathematical finance, the discretization error corresponds to the discrete hedging error, which is inevitably associated with the discretization of hedging strategies. The limit distribution tells us how to quantify the risk of hedging by finite transactions that is usually negleted in continuous-time financial modeling.

There are two directions for a refinement of the central limit theorem; the large deviation theory and the theory of the Edgeworth expansion. The former focuses the tail probability and gives a precise asymptotic formula in terms of the so-called rate function. The latter focuses the behavior around the mean and gives an expansion formula in terms of Hermite polynomials with coefficients determined by moments. The former tends to give a more precise approximation, while it requires to compute the rate function that is impossible in many problems. The latter is often less precise; however it only requires to compute moments that is usually feasible. Further, in the context of statistics, the Edgeworth expansion provides a theoretical justification of some popular computational techniques like the bootstrap; see Hall [5]. Unfortunaly, both of the refinements are not directly applicable to the discrezation error of Itô integral because the limit distribution is in general not normal but only mixed normal.

The theory of the Edgeworth expansion in mixed normal limit has recently developed by Yoshida [12] by extending his martingale expansion approach [10, 11] in normal limit. It turns out that the expansion formula involves entangled anticipating effects described in terms of the Malliavin calculus. The Yoshida theory provides a general framework and for each of concrete applications, a non-negligble effort is still required to obtain an explicit expression of the expansion terms. An application to the power variation of diffusion processes is given by Podolskij and Yoshida [8].

The aim of this study is to give an explicit expansion formula for the regular discretization error of Itô integrals. We treat a far more restricted problem than in Yoshida [12] and try to get a more explicit formula. For this purpose, instead of just analyzing abstract terms given in Yoshida [12], we introduce a 
more systematic approach based on the anticipating Itô formula, developed by Nualart and Pardoux in [7]. This alternative approach is in a sense more elementary and the appearance of Hermite polynomials is more natural.

\section{Anticipating stochastic calculus}

In this section we recall the basic results on the Malliavin calculus and the anticipating stochastic calculus we use through the paper. For a more detailed introduction to this subject we refer to [6].

Let $T>0$ and $\left(\Omega, \mathcal{F}, P,\left\{\mathcal{F}_{t}\right\}_{0 \leq t \leq T}\right)$ be an filtered probability space satisfying the usual conditions which supports a standard Brownian motion $W$ on $[0, T]$. We denote by $E$ the expectation operator with respect to $P$, and denote by $D$ the Malliavin derivative operator with respect to $W$. More precisely, we will assume a partial Malliavin structure $(\Omega, \mathcal{F}, P)=\left(\Omega_{1} \times \Omega_{2}, \mathcal{F}_{1} \otimes \mathcal{F}_{2}, P_{1} \otimes P_{2}\right)$ with $\left(\Omega_{1}, \mathcal{F}_{1}, P_{1}\right)$ being the Wiener space associated with $W$. It is well-known that $D$ is a closable operator from $L^{p}(\Omega)$ to $L^{p}([0, T] \times \Omega)$, for any $p \geq 1$. We will denote by $\mathbb{D}^{1, p}$ the domain of $D$ in $L^{p}(\Omega)$. We also consider the iterated derivatives $D^{n}$, for $n \geq 1$, whose domains will be denoted by $\mathbb{D}^{n, p}$. The Sobolev norm of $\mathbb{D}^{n, p}$ will be denoted by $\|\cdot\|_{n, p}$. We will use the notation $\mathbb{L}^{n, p}=L^{2}\left([0, T] ; \mathbb{D}^{n, p}\right)$.

Given a process $X \in \mathbb{L}^{1, p}, D^{+} X$ and $D^{-} X$ will be the element of $L^{p}([0, T] \times \Omega)$ satisfying

$$
\lim _{n \rightarrow \infty} \int_{0}^{T} \sup _{s<t \leq\left(s+\frac{1}{n}\right) \wedge T} E\left[\left|D_{s} X_{t}-D^{+} X_{t}\right|^{p}\right] \mathrm{d} t=0
$$

and

$$
\lim _{n \rightarrow \infty} \int_{0}^{T} \sup _{\left(s-\frac{1}{n}\right) \vee 0 \leq t<s} E\left[\left|D_{s} X_{t}-D^{-} X_{t}\right|^{p}\right] \mathrm{d} t=0
$$

respectively. Moreover, we will denote by $\mathbb{L}_{+}^{1, p}$ and $\mathbb{L}_{-}^{1, p}$ the sets of such processes $X$ in $\mathbb{L}^{1, p}$ that admit $D^{+} X$ and $D^{-} X$ with (1) and (2) respectively.

The adjoint of the derivative operator $D$, denoted by $\delta$, is an extension of the Itô integral in the sense that the set $L_{a}^{2}([0, T] \times \Omega)$ of square integrable and $\left\{\mathcal{F}_{t}\right\}$ adapted processes is included in the domain of $\delta$ and the operator $\delta$ restricted to $L_{a}^{2}([0, T] \times \Omega)$ coincides with the Itô integral. We will make use of the notation

$$
\delta(u)=\int_{0}^{T} u_{t} \mathrm{~d} W_{t}
$$

We recall that $\mathbb{L}^{n, 2}$ is included in the domain of $\delta$ for all $n \geq 1$.

Proposition 2.1 (The Malliavin derivative of an Itô process) Consider an Itô process of the form

$$
S_{t}=S_{0}+\int_{0}^{t} S_{u}^{\prime} \mathrm{d} u+\int_{0}^{t} S_{u}^{\prime \prime} \mathrm{d} W_{u}
$$


where $S_{0}$ is a positive constant and $S^{\prime}, S^{\prime \prime}$ are adapted processes in $\mathbb{L}^{1,2}$. Then $S \in \mathbb{L}^{1,2}$ and for all $0<r<t<T$,

$$
D_{r} S_{t}=\int_{r}^{t} D_{r} S_{u}^{\prime} \mathrm{d} u+S_{r}^{\prime \prime}+\int_{r}^{t} D_{r} S_{u}^{\prime \prime} \mathrm{d} W_{u} .
$$

The key tool for this work is the following anticipating Itô formula; see Theorem 3.2.4 of [6].

Theorem 2.1 Let $S$ and $A$ be processes of the form

$$
\begin{aligned}
& S_{t}=S_{0}+\int_{0}^{t} S_{u}^{\prime} \mathrm{d} u+\int_{0}^{t} S_{u}^{\prime \prime} \mathrm{d} W_{u}, \\
& A_{t}=\int_{t}^{T} A_{s}^{\prime} \mathrm{d} s,
\end{aligned}
$$

where $S^{\prime}, S^{\prime \prime} \in L_{a}^{2}([0, T] \times \Omega)$ and $A^{\prime} \in \mathbb{L}^{1,2}$. Then, $A \in \mathbb{L}_{-}^{1,2}$,

$$
D^{-} A_{u}=\int_{u}^{T} D_{u} A_{s}^{\prime} \mathrm{d} s
$$

and for any $f \in C_{b}^{2}\left(\mathbb{R}^{2}\right)$ with

$$
E\left[\left(\int_{0}^{T}\left|f_{1,1}\left(A_{u}, S_{u}\right) D^{-} A_{u}\right| \mathrm{d}\langle S, W\rangle_{u}\right)^{2}\right]<\infty,
$$

it holds

$$
\begin{aligned}
f\left(A_{t}, S_{t}\right)= & f\left(A_{0}, S_{0}\right)+\int_{0}^{t} f_{1,0}\left(A_{u}, S_{u}\right) \mathrm{d} A_{u}+\int_{0}^{t} f_{0,1}\left(A_{u}, S_{u}\right) \mathrm{d} S_{u} \\
& +\int_{0}^{t} f_{1,1}\left(A_{u}, S_{u}\right) D^{-} A_{u} \mathrm{~d}\langle S, W\rangle_{u}+\frac{1}{2} \int_{0}^{t} f_{0,2}\left(A_{u}, S_{u}\right) \mathrm{d}\langle S\rangle_{u}
\end{aligned}
$$

for all $t \in[0, T]$, where

$$
\int_{0}^{t} f_{1,0}\left(A_{u}, S_{u}\right) \mathrm{d} S_{u}=\int_{0}^{t} f_{1,0}\left(A_{u}, S_{u}\right) S_{u}^{\prime} \mathrm{d} u+\delta\left(1_{[0, t]} f_{1,0}(A, S) S^{\prime \prime}\right) .
$$

\section{The statement of the main result}

We consider the limit distribution of a normalized regular discretization error of a stochastic integral

$$
Z_{t}^{n}=\sqrt{n}\left\{\int_{0}^{t} X_{s} \mathrm{~d} Y_{s}-\sum_{j=0}^{\infty} X_{t_{j}^{n}}\left(Y_{t_{j+1}^{n} \wedge t}-Y_{t_{j}^{n} \wedge t}\right)\right\}
$$


as $n \rightarrow \infty$, where $t_{j}^{n}=j / n$ and $X$ and $Y$ are continuous Itô processes of the form

$$
\mathrm{d} X_{t}=\Xi_{t} \mathrm{~d} t+\Gamma_{t} \mathrm{~d} W_{t}, \quad \mathrm{~d} Y_{t}=\Theta_{t} \mathrm{~d} t+\Sigma_{t} \mathrm{~d} W_{t},
$$

where $\Xi, \Theta, \Gamma, \Sigma$ are continuous processes adapted to the filtration $\left\{\mathcal{F}_{t}\right\}$. We further assume that the product $\Gamma \Sigma$ is not identically zero and that $\Gamma$ and $\Sigma^{2}$ (the square of $\Sigma$ ) are also Itô processes of the form

$$
\mathrm{d} \Gamma_{t}=\check{\Gamma}_{t} \mathrm{~d} t+\hat{\Gamma}_{t} \mathrm{~d} W_{t}, \mathrm{~d} \Sigma_{t}^{2}=\check{\Sigma}_{t} \mathrm{~d} t+\hat{\Sigma}_{t} \mathrm{~d} W_{t},
$$

where $\check{\Gamma}, \check{\Sigma}, \hat{\Gamma}$ and $\hat{\Sigma}$ are adapted processes.

We will assume the following hypotheses:

(H1) For all $p>0$, the adapted processes $\Xi, \Theta, \check{\Gamma}, \hat{\Gamma}, \check{\Sigma}, \hat{\Sigma}$ are in $\mathbb{L}^{4, p}$.

(H2) $\Theta$ and $\Sigma$ belong to $\mathbb{L}_{+}^{1, p}$ for all $p>2$ and $D^{+} \Theta$ and $D^{+} \Sigma$ are continuous.

(H3) For any $U \in\{\Xi, \Theta, \hat{\Sigma}, \hat{\Gamma}\}$,

$$
\sup _{t \in[0, T]} E\left[\left|U_{t}\right|^{4}\right]<\infty, \lim _{n \rightarrow \infty} \sup _{|s-t| \leq \frac{1}{n}} E\left[\left|U_{t}-U_{s}\right|^{2}\right]=0 .
$$

Remark 3.1 All of the above assumptions are satisfied, for example, if $X_{t}=g_{X}\left(t, W_{t}\right)$ and $Y_{t}=g_{Y}\left(t, W_{t}\right)$ for some $C^{\infty}$ functions $g_{X}, g_{Y}$ with all the derivatives being of at most exponential growth. This is the case of discrete hedging for a European $\mathrm{C}^{\infty}$ payoff of at most polynomial growth under the Black-Scholes model.

Remark 3.2 Under (H1), $\Gamma, \Sigma^{2}, X$ and $Y$ are adapted processes in $\mathbb{L}^{4, p}$ for all $p>0$. Moreover, for all $p>0$,

$$
\sup _{t \in[0, T]} E\left[\left|\Gamma_{t}\right|^{p}\right]+\sup _{t \in[0, T]} E\left[\left|\Sigma_{t}\right|^{p}\right]<\infty .
$$

We will also make use of the following notation

$$
V_{t}=\frac{1}{2} \int_{t}^{T} \Gamma_{s}^{2} \Sigma_{s}^{2} \mathrm{~d} s
$$

Using Proposition 2.1, it is easy to see that $V \in \mathbb{L}_{-}^{1, p}$ for any $p>1$ and

$$
D^{-} V_{t}=\frac{1}{2} \int_{t}^{T} D_{t}\left[\Gamma_{s}^{2} \Sigma_{s}^{2}\right] \mathrm{d} s
$$

Moreover, $D^{-} V$ and $D\left[\Gamma_{s}^{2} \Sigma_{s}^{2}\right] \in \mathbb{L}_{-}^{1, p}$ for any $s$.

Now we can state the main result of this paper. 
Theorem 3.1 Under (H1), (H2) and (H3), for any $f \in C_{b}^{\infty}(\mathbb{R})$, we have

$$
E\left[f\left(Z_{T}^{n}\right)\right]=\int_{\mathbb{R}} f(z) E\left[Q_{n}(z)\right] \mathrm{d} z+o\left(n^{-1 / 2}\right),
$$

where

$$
\begin{gathered}
Q_{n}(z)=\left\{1+\frac{1}{\sqrt{n}}\left\{A_{1} H_{1}\left(z, V_{0}\right)+A_{3} H_{3}\left(z, V_{0}\right)+A_{5} H_{5}\left(z, V_{0}\right)\right\}\right\} \phi\left(z, V_{0}\right), \\
H_{k}(z, t)=\frac{(-1)^{k}}{\phi(z, t)} \frac{\partial^{k}}{\partial z^{k}} \phi(z, t), k=1,3,5, \\
\phi(z, t)=\frac{1}{\sqrt{2 \pi t}} \exp \left\{-\frac{z^{2}}{2 t}\right\}
\end{gathered}
$$

and

$$
\begin{aligned}
& A_{1}=\frac{1}{2} \int_{0}^{T}\left(\Xi_{t} \Theta_{t}+D^{+} \Theta_{t} \Gamma_{t}\right) \mathrm{d} t \\
& A_{3}=\frac{1}{4} \int_{0}^{T}\left(\left(\Xi_{t} \Sigma_{t}+\Theta_{t} \Gamma_{t}+D^{+} \Sigma_{t} \Gamma_{t}\right) D^{-} V_{t}+\Gamma_{t} \Sigma_{t}\left(D^{-}\right)^{2} V_{t}\right) \mathrm{d} t+\frac{1}{6} \int_{0}^{T} \Sigma_{t}^{3} \Gamma_{t}^{3} \mathrm{~d} t \\
& A_{5}=\frac{1}{8} \int_{0}^{T} \Gamma_{t} \Sigma_{t}\left|D^{-} V_{t}\right|^{2} \mathrm{~d} t .
\end{aligned}
$$

This is an Edgeworth type expansion in that the expansion coefficients are written in terms of the normal density and Hermite polynomials. An important difference from the classical Edgeworth expansion formula is that the limit variance $V_{0}$ is random. Due to this anticipating feature, the 5 th order Hermite polynomial appears in this first order expansion, while Hermite polynomials of only up to 3rd order appear in the first order Edgeworth expansion in the classical situation, that is, where $V_{0}$ is deterministic. The 1st and 3rd order Hermite polynomial terms represent, respectively, the bias and the skewness of the limit distribution of $O\left(n^{-1 / 2}\right)$.

\section{The outline of the proof}

Let $X_{t}^{n}=X_{[n t] / n}$ and

$$
V_{t}^{n}=n \int_{t}^{T}\left(X_{s}-X_{s}^{n}\right)^{2} \Sigma_{s}^{2} \mathrm{~d} s
$$

Then we have

$$
Z_{t}^{n}=\sqrt{n} \int_{0}^{t}\left(X_{s}-X_{s}^{n}\right) \mathrm{d} Y_{s}, \mathrm{~d}\left\langle Z^{n}\right\rangle_{t}=-\mathrm{d} V_{t}^{n}
$$


For $f \in C_{b}^{\infty}(\mathbb{R})$, define

$$
q(t, x)=\int f(z) \phi(x-z, t) \mathrm{d} z
$$

Note that $q$ is the solution of the heat equation

$$
q_{1,0}=\frac{1}{2} q_{0,2}, \quad q(0, x)=f(x),
$$

which in particular implies that $q \in C_{b}^{\infty}([0, \infty) \times \mathbb{R})$.

By (5), (6),(H1), (H2), (H3) and Theorem 2.1,

$$
\begin{aligned}
E\left[f\left(Z_{T}^{n}\right)\right]=E\left[q\left(V_{T}^{n}, Z_{T}^{n}\right)\right] & \\
=E\left[q\left(V_{0}^{n}, 0\right)\right] & +\sqrt{n} \int_{0}^{T} E\left[q_{0,1}\left(V_{t}^{n}, Z_{t}^{n}\right)\left(X_{t}-X_{t}^{n}\right) \Theta_{t} \mathrm{~d} t\right] \\
& +\sqrt{n} \int_{0}^{T} E\left[q_{1,1}\left(V_{t}^{n}, Z_{t}^{n}\right) D^{-} V_{t}^{n}\left(X_{t}-X_{t}^{n}\right) \sum_{t}\right] \mathrm{d} t,
\end{aligned}
$$

where

We will show in Lemma 6.1 that

$$
D^{-} V_{t}^{n}=n \int_{t}^{T} D_{t}\left[\left(X_{s}-X_{s}^{n}\right)^{2} \Sigma_{s}^{2}\right] \mathrm{d} s .
$$

$$
E\left[q\left(V_{0}^{n}, 0\right)\right]=E\left[q\left(V_{0}, 0\right)\right]+o\left(n^{-1 / 2}\right),
$$

in Lemma 6.2 that

$$
\begin{aligned}
& n \int_{0}^{T} E\left[q_{0,1}\left(V_{t}^{n}, Z_{t}^{n}\right)\left(X_{t}-X_{t}^{n}\right) \Theta_{t}\right] \mathrm{d} t \\
& \rightarrow \frac{1}{2} \int_{0}^{T} E\left[q_{0,1}\left(V_{0}, 0\right)\left(\Xi_{t} \Theta_{t}+D^{+} \Theta_{t} \Gamma_{t}\right)\right] \mathrm{d} t+\frac{1}{2} \int_{0}^{T} E\left[q_{1,1}\left(V_{0}, 0\right) D^{-} V_{t} \Theta_{t} \Gamma_{t}\right] \mathrm{d} t \\
& =E\left[q_{0,1}\left(V_{0}, 0\right) A_{1}\right]+\frac{1}{4} E\left[q_{0,3}\left(V_{0}, 0\right) \int_{0}^{T} D^{-} V_{t} \Theta_{t} \Gamma_{t}\right] \mathrm{d} t
\end{aligned}
$$

and in Lemma 6.3 that

$$
\begin{aligned}
& n \int_{0}^{T} E\left[q_{1,1}\left(V_{t}^{n}, Z_{t}^{n}\right) D^{-} V_{t}^{n}\left(X_{t}-X_{t}^{n}\right) \Sigma_{t}\right] \mathrm{d} t \\
& \rightarrow \frac{1}{2} E\left[q_{1,1}\left(V_{0}, 0\right) \int_{0}^{T}\left(D^{-} V_{t} \Xi_{t} \Sigma_{t}+\left(D^{-}\right)^{2} V_{t} \Sigma_{t} \Gamma_{t}+D^{-} V_{t} D^{+} \Sigma_{t} \Gamma_{t}\right) \mathrm{d} t\right] \\
& \left.\quad+\frac{1}{2} E\left[q_{2,1}\left(V_{0}, 0\right) \int_{0}^{T}\left|D^{-} V_{t}\right|^{2} \Gamma_{t} \Sigma_{t}\right] \mathrm{d} t\right]+\frac{1}{3} E\left[q_{1,1}\left(V_{0}, 0\right) \int_{0}^{T} \Sigma_{t}^{3} \Gamma_{t}^{3} d t\right] \\
& =\frac{1}{4} E\left[q_{0,3}\left(V_{0}, 0\right) \int_{0}^{T}\left(D^{-} V_{t} \Xi_{t} \Sigma_{t}+\left(D^{-}\right)^{2} V_{t} \Sigma_{t} \Gamma_{t}+D_{t}^{-} V_{t} D^{+} \Sigma_{t} \Gamma_{t}+\frac{2}{3} \Sigma_{t}^{3} \Gamma_{t}^{3}\right) \mathrm{d} t\right] \\
& \quad+E\left[q_{0,5}\left(V_{0}, 0\right) A_{5}\right] .
\end{aligned}
$$


Since

$$
q_{0, k}(t, x)=\int_{\mathbb{R}} f(z) \frac{\partial^{k}}{\partial x^{k}} \phi(x-z, t) \mathrm{d} z=\int_{\mathbb{R}} f(z) H_{k}(z-x, t) \phi(z-x, t) \mathrm{d} z
$$

for $k=0,1,2, \ldots$, the expansion claimed in Theorem 3.1 follows.

\section{The approximating processes}

This section is devoted to prove some results of the approximating processes $V^{n}$ and $Z^{n}$ that we will use through the paper. We recall the following lemma, which will be repeatedly used in the sequel:

Lemma 5.1 Let $p \geq 1, p^{\prime}=p /(p-1), F \in L^{p}([0, T] \times \Omega)$ and $G \in L^{p^{\prime}}([0, T] \times \Omega)$. Denote their norms as

$$
\|F\|_{p}=\left\{\int_{0}^{T} E\left[\left|F_{t}\right|^{p}\right] \mathrm{d} t\right\}^{1 / p},\|G\|_{p^{\prime}}=\left\{\int_{0}^{T} E\left[\left|G_{t}\right|^{p \prime}\right] \mathrm{d} t\right\}^{1 / p^{\prime}} .
$$

Then, for all $n \in \mathbb{N}$,

$$
E\left[n \int_{0}^{T} \int_{[n t] / n}^{t}\left|F_{s} G_{t}\right| \mathrm{d} s \mathrm{~d} t\right] \leq\|F\|_{p}\|G\|_{p^{\prime}} .
$$

Proof: By Hölder's inequality,

$$
E\left[n \int_{0}^{T} \int_{[n t] / n}^{t}\left|F_{s} G_{t}\right| \mathrm{d} \mathrm{d} \mathrm{d} t\right] \leq\|G\|_{p^{\prime}} E\left[\int_{0}^{T}\left|n \int_{[n t] / n}^{t}\right| F_{s}|\mathrm{~d} s|^{p} \mathrm{~d} t\right]^{1 / p} .
$$

Since $n(t-[n t] / n) \leq 1$, by Jensen's inequality,

$$
E\left[\int_{0}^{T}\left|n \int_{[n t] / n}^{t}\right| F_{s}|\mathrm{~d} s|^{p} \mathrm{~d} t\right] \leq E\left[\int_{0}^{T} n \int_{[n t] / n}^{t}\left|F_{s}\right|^{p} \mathrm{~d} s \mathrm{~d} t\right] .
$$

Since $[a] \leq b$ if and only if $a<[b]+1$ in general for $a, b \geq 0,[n t] / n \leq s \leq t$ is equivalent to $s \leq t<([n s]+1) / n$. Therefore by the Fubini theorem,

$$
E\left[\int_{0}^{T} n \int_{[n t] / n}^{t}\left|F_{s}\right|^{p} \mathrm{~d} s \mathrm{~d} t\right]=E\left[\int_{0}^{T}(1+[n s]-n s)\left|F_{s}\right|^{p} \mathrm{~d} s\right] \leq\|F\|_{p}^{p} .
$$

Lemma 5.2 Under (H1) and (H3)

$$
\sup _{\left|s-s^{\prime}\right|<\delta}\left\|\Sigma_{s}^{2}-\Sigma_{s^{\prime}}^{2}\right\|_{1,4} \rightarrow 0
$$

and

as $\delta \rightarrow 0$

$$
\sup _{\left|s-s^{\prime}\right|<\delta}\left\|\Sigma_{s}^{2} \Gamma_{s}^{2}-\Sigma_{s^{\prime}}^{2} \Gamma_{s^{\prime}}^{2}\right\|_{1,4} \rightarrow 0
$$


Proof: For the sake of simplicity we can assume that $s>s^{\prime}$. Using (H1) we can see that

$$
D_{t} \Sigma_{s}^{2}-D_{t} \Sigma_{s^{\prime}}^{2}=\int_{s^{\prime}}^{s} D_{t} \check{\Sigma}_{r}+\hat{\Sigma}_{t} \mathbf{1}_{\left[s^{\prime}, s\right]}(t)+\int_{s^{\prime}}^{s} D_{t} \hat{\Sigma}_{r} d W_{r} .
$$

Then, (H3) allows us to show the first convergence. The second convergence is treated similarly.

\subsection{The stable convergence of $V^{n}$}

The goal of this subsection is to prove Proposition 5.1 below, which is necessary to prove Lemmas 6.1 and 6.2 in Section 6 .

Lemma 5.3 Let us consider two processes $\Sigma$ and $\Gamma$ defined as in (4). Then, under (H1),

$$
\sqrt{n}\left(\int_{0}^{T}\left(\Gamma_{t}^{2} \Sigma_{t}^{2}-\Gamma_{[n t] / n}^{2} \Sigma_{[n t] / n}^{2}\right) \mathrm{d} t\right)
$$

converges to 0 in $L^{1}$ as $n \rightarrow \infty$.

Proof: By the assumption on $\Gamma$ and $\Sigma, \Gamma^{2} \Sigma^{2}$ is an Itô semimartingale and so, of the form

$$
\mathrm{d}\left(\Gamma^{2} \Sigma^{2}\right)_{t}=\alpha_{t} \mathrm{~d} t+\beta_{t} \mathrm{~d} W_{t}
$$

with $\alpha, \beta \in L_{a}^{2}([0, T] \times \Omega)$. By Lemma5.1.

$$
\sqrt{n} \int_{0}^{T} \int_{[n t] / n}^{t} \alpha_{s} \mathrm{~d} s \mathrm{~d} t \rightarrow 0
$$

in $L^{1}$. Further, denoting $t_{j}=t_{j}^{n} \wedge T$,

$$
\begin{aligned}
n E\left[\left|\sum_{j} \int_{t_{j}}^{t_{j+1}} \int_{t_{j}}^{t} \beta_{s} \mathrm{~d} W_{s} \mathrm{~d} t\right|^{2}\right] & =n \sum_{j} E\left[\left|\int_{t_{j}}^{t_{j+1}} \int_{t_{j}}^{t} \beta_{s} \mathrm{~d} W_{s} \mathrm{~d} t\right|^{2}\right] \\
& =n \sum_{j} E\left[\int_{t_{j}}^{t_{j+1}} \int_{t_{j}}^{t_{j+1}} \int_{t_{j}}^{t} \beta_{s} \mathrm{~d} W_{s} \int_{t_{j}}^{u} \beta_{v} \mathrm{~d} W_{v} \mathrm{~d} u \mathrm{~d} t\right] \\
& =2 n \sum_{j} \int_{t_{j}}^{t_{j+1}} \int_{t_{j}}^{t} \int_{t_{j}}^{u} E\left[\beta_{v}^{2}\right] \mathrm{d} v \mathrm{~d} u \mathrm{~d} t \\
& =2 n \int_{0}^{T} \int_{[n t] / n}^{t} \int_{[n t] / n}^{u} E\left[\beta_{v}^{2}\right] \mathrm{d} v \mathrm{~d} u \mathrm{~d} t \\
& =2 n \int_{0}^{T} \int_{[n t]] / n}^{t}(t-v) E\left[\beta_{v}^{2}\right] \mathrm{d} v \mathrm{~d} t \\
& \leq 2 \int_{0}^{T} \int_{[n t] / n}^{t} E\left[\beta_{v}^{2}\right] \mathrm{d} v \mathrm{~d} t \rightarrow 0
\end{aligned}
$$

by Lemma 5.1, which completes the proof. 
Proposition 5.1 Assume that hypotheses (H1) and (H3) hold. Then $\sqrt{n}\left(V_{0}^{n}-V_{0}\right)$ is uniformly integrable and

$$
\sqrt{n}\left(V_{0}^{n}-V_{0}\right) \rightarrow \mathcal{M N}\left(0, \frac{1}{3} \int_{0}^{T} \Gamma_{t}^{4} \Sigma_{t}^{4} \mathrm{~d} t\right)
$$

stably as $n \rightarrow \infty$. In particular, for any uniformly bounded random variables $U_{n}, U$ such that $U_{n} \rightarrow U$ in probability,

$$
E\left[\sqrt{n}\left(V_{0}^{n}-V_{0}\right) U_{n}\right] \rightarrow 0 .
$$

Proof: We will denote $t_{j}=t_{j}^{n} \wedge T$ for brevity. Denote by $E_{t_{j}}$ the conditional expectation given $\mathcal{F}_{t_{j}}$. We will use $C$ as a generic constant.

$$
\begin{aligned}
V_{0}^{n}-V_{0}= & n \int_{0}^{T}\left(X_{t}-X_{t}^{n}\right)^{2} \Sigma_{t}^{2} \mathrm{~d} t-\frac{1}{2} \int_{0}^{T} \Gamma_{t}^{2} \Sigma_{t}^{2} \mathrm{~d} t \\
= & n \sum_{j} \int_{t_{j}}^{t_{j+1}}\left(\int_{t_{j}}^{t} \Xi_{u} \mathrm{~d} u\right)^{2} \Sigma_{t}^{2} \mathrm{~d} t+2 n \sum_{j} \int_{t_{j}}^{t_{j+1}} \int_{t_{j}}^{t} \Xi_{u} \mathrm{~d} u \int_{t_{j}}^{t} \Gamma_{u} \mathrm{~d} W_{u} \Sigma_{t}^{2} \mathrm{~d} t \\
& +n \sum_{j} \int_{t_{j}}^{t_{j+1}}\left(\Gamma_{t_{j}} \int_{t_{j}}^{t} \mathrm{~d} W_{u}+\int_{t_{j}}^{t}\left(\Gamma_{u}-\Gamma_{t_{j}}\right) \mathrm{d} W_{u}\right)^{2} \Sigma_{t}^{2} \mathrm{~d} t-\frac{1}{2} \int_{0}^{1} \Gamma_{t}^{2} \Sigma_{t}^{2} \mathrm{~d} t
\end{aligned}
$$

The sum of the last two terms can be written as

$$
\begin{aligned}
& n \sum_{j} \Gamma_{t_{j}}^{2} \Sigma_{t_{j}}^{2} \int_{t_{j}}^{t_{j+1}}\left(W_{t}-W_{t_{j}}\right)^{2} \mathrm{~d} t-\frac{1}{2} \int_{0}^{1} \Gamma_{t}^{2} \Sigma_{t}^{2} \mathrm{~d} t \\
& +n \sum_{j} \Gamma_{t_{j}}^{2} \int_{t_{j}}^{t_{j+1}}\left(W_{t}-W_{t_{j}}\right)^{2}\left(\Sigma_{t}^{2}-\Sigma_{t_{j}}^{2}\right) \mathrm{d} t \\
& +2 n \sum_{j} \Gamma_{t_{j}} \int_{t_{j}}^{t_{j+1}}\left(W_{t}-W_{t_{j}}\right) \int_{t_{j}}^{t}\left(\Gamma_{u}-\Gamma_{t_{j}}\right) \mathrm{d} W_{u} \Sigma_{t}^{2} \mathrm{~d} t \\
& +n \sum_{j} \int_{t_{j}}^{t_{j+1}}\left(\int_{t_{j}}^{t}\left(\Gamma_{u}-\Gamma_{t_{j}}\right) \mathrm{d} W_{u}\right)^{2} \Sigma_{t}^{2} \mathrm{~d} t .
\end{aligned}
$$

Step 1). First we show

$$
\begin{aligned}
& \sqrt{n}\left(n \sum_{j} \Gamma_{t_{j}}^{2} \Sigma_{t_{j}}^{2} \int_{t_{j}}^{t_{j+1}}\left(W_{t}-W_{t_{j}}\right)^{2} \mathrm{~d} t-\frac{1}{2} \int_{0}^{1} \Gamma_{t}^{2} \Sigma_{t}^{2} \mathrm{~d} t\right) \\
& \rightarrow \mathcal{M N}\left(0, \frac{1}{3} \int_{0}^{1} \Gamma_{t}^{4} \Sigma_{t}^{4} \mathrm{~d} t\right) .
\end{aligned}
$$

By Lemma 5.3, it suffices to show

$$
\sqrt{n} \sum_{j} \Gamma_{t_{j}}^{2} \Sigma_{t_{j}}^{2}\left(\int_{t_{j}}^{t_{j+1}} n\left(W_{t}-W_{t_{j}}\right)^{2} \mathrm{~d} t-\frac{1}{2 n}\right) \rightarrow \mathcal{M N}\left(0, \frac{1}{3} \int_{0}^{1} \Gamma_{t}^{4} \Sigma_{t}^{4} \mathrm{~d} t\right) .
$$


The left hand side is equal to

$$
2 n^{3 / 2} \sum_{j} \Gamma_{t_{j}}^{2} \Sigma_{t_{j}}^{2} \int_{t_{j}}^{t_{j+1}} \int_{t_{j}}^{t}\left(W_{u}-W_{t_{j}}\right) \mathrm{d} W_{u} \mathrm{~d} t=: \sum_{j} A_{j} .
$$

Since

$$
E_{t_{j}}\left[A_{j}\right]=E_{t_{j}}\left[A_{j}\left(W_{t_{j+1}}-W_{t_{j}}\right)\right]=0
$$

and

$$
E_{t_{j}}\left[A_{j}^{2}\right]=\frac{1}{3 n} \Gamma_{t_{j}}^{4} \Sigma_{t_{j}}^{4}
$$

the result follows from Jacod's theorem of stable convergence. From this computation, the uniform integrability is also clear.

Step 2). Next, we show

$$
n^{3 / 2} \sum_{j} \Gamma_{t_{j}}^{2} \int_{t_{j}}^{t_{j+1}}\left(W_{t}-W_{t_{j}}\right)^{2}\left(\Sigma_{t}^{2}-\Sigma_{t_{j}}^{2}\right) \mathrm{d} t
$$

is uniformly integrable and converges to 0 in probability. The boundedness in $L^{2}$ is not difficult to see, from which the uniform integrability follows. Since

$$
E\left[\left|\int_{t_{j}}^{t_{j+1}}\left(W_{t}-W_{t_{j}}\right)^{2} \int_{t_{j}}^{t} \check{\Sigma}_{u} \mathrm{~d} u \mathrm{~d} t\right|\right] \leq n^{-3 / 2} \sqrt{E\left[\int_{t_{j}}^{t_{j+1}}\left(\int_{t_{j}}^{t} \check{\Gamma}_{u} \mathrm{~d} u\right)^{2} \mathrm{~d} t\right]}
$$

by the Cauchy-Schwarz inequality, we have

$$
E\left[\left|n^{3 / 2} \sum_{j} \Gamma_{t_{j}}^{2} \int_{t_{j}}^{t_{j+1}}\left(W_{t}-W_{t_{j}}\right)^{2} \int_{t_{j}}^{t} \check{\Sigma}_{u} \mathrm{~d} u \mathrm{~d} t\right|\right] \leq \frac{\|\check{\Sigma}\|_{2}}{\sqrt{n}} \rightarrow 0 .
$$

Therefore, it suffices to show

$$
n^{3 / 2} \sum_{j} \Gamma_{t_{j}}^{2} \int_{t_{j}}^{t_{j+1}}\left(W_{t}-W_{t_{j}}\right)^{2} \int_{t_{j}}^{t} \hat{\Sigma}_{u} \mathrm{~d} W_{u} \mathrm{~d} t \rightarrow 0
$$

in probability. To see this, note that

$$
\begin{aligned}
& E\left[\left|n^{3 / 2} \sum_{j} \Gamma_{t_{j}}^{2} \hat{\Sigma}_{t_{j}} \int_{t_{j}}^{t_{j+1}}\left(W_{t}-W_{t_{j}}\right)^{3} \mathrm{~d} t\right|^{2}\right] \\
& \leq C n^{3} E\left[\sum_{j} \Gamma_{t_{j}}^{4} \hat{\Sigma}_{t_{j}}^{2} E_{t_{j}}\left[\left(\int_{t_{j}}^{t_{j+1}}\left(W_{t}-W_{t_{j}}\right)^{3} \mathrm{~d} t\right)^{2}\right]\right] \leq \frac{C}{n}
\end{aligned}
$$


by (H1) and (H3). Therefore, with the aid of (H3), it follows from

$$
\begin{aligned}
& E\left[\left|\sum_{j} \Gamma_{t_{j}}^{2} \int_{t_{j}}^{t_{j+1}}\left(W_{t}-W_{t_{j}}\right)^{2} \int_{t_{j}}^{t}\left(\hat{\Sigma}_{u}-\hat{\Sigma}_{t_{j}}\right) \mathrm{d} W_{u} \mathrm{~d} t\right|\right] \\
& =E\left[\left|\int_{0}^{T} \Gamma_{[n t] / n}\left(W_{t}-W_{[n t] / n}\right)^{2} \int_{[n t] / n}^{t}\left(\hat{\Sigma}_{u}-\hat{\Sigma}_{[n t] / n}\right) \mathrm{d} W_{u} \mathrm{~d} t\right|\right] \\
& \leq E\left[\int_{0}^{T} \Gamma_{[n t] / n}^{4} \mathrm{~d} t\right]^{1 / 4} E\left[\int_{0}^{T}\left|W_{t}-W_{[n t] / n}\right|^{8} \mathrm{~d} t\right]^{1 / 4} E\left[\int_{0}^{T} \int_{[n t] / n}^{t}\left|\hat{\Sigma}_{u}-\hat{\Sigma}_{[n t] / n}\right|^{2} \mathrm{~d} u\right]^{1 / 2} \\
& \leq C n^{-3 / 2} \sup _{0 \leq|s-t| \leq 1 / n} E\left[\left|\hat{\Sigma}_{s}-\hat{\Sigma}_{t}\right|^{2}\right]^{1 / 2}=o\left(n^{-3 / 2}\right) .
\end{aligned}
$$

Step 3). Next, we look at

$$
2 n^{3 / 2} \sum_{j} \Gamma_{t_{j}} \int_{t_{j}}^{t_{j+1}}\left(W_{t}-W_{t_{j}}\right) \int_{t_{j}}^{t}\left(\Gamma_{u}-\Gamma_{t_{j}}\right) \mathrm{d} W_{u} \Sigma_{t}^{2} \mathrm{~d} t .
$$

By a similar argument to the above, the problem reduces to showing that

$$
2 n^{3 / 2} \sum_{j} \Gamma_{t_{j}} \Sigma_{t_{j}}^{2} \int_{t_{j}}^{t_{j+1}}\left(W_{t}-W_{t_{j}}\right) \int_{t_{j}}^{t} \int_{t_{j}}^{u} \hat{\Gamma}_{s} \mathrm{~d} W_{s} \mathrm{~d} W_{u} \mathrm{~d} t
$$

converges to 0 in $L^{2}$, where $\hat{\Gamma}$ is the diffusion coefficient of the continuous Itô process $\Gamma$. Since

$$
\begin{aligned}
& E_{t_{j}}\left[\int_{t_{j}}^{t_{j+1}}\left(W_{t}-W_{t_{j}}\right) \int_{t_{j}}^{t} \int_{t_{j}}^{u} \hat{\Gamma}_{s} \mathrm{~d} W_{s} \mathrm{~d} W_{u} \mathrm{~d} t\right] \\
& =\int_{t_{j}}^{t_{j+1}} E_{t_{j}}\left[\int_{t_{j}}^{t} \int_{t_{j}}^{u} \hat{\Gamma}_{s} \mathrm{~d} W_{s} \mathrm{~d} u\right] \mathrm{d} t=0,
\end{aligned}
$$


we have

$$
\begin{aligned}
& E\left[\left|2 n^{3 / 2} \sum_{j} \Gamma_{t_{j}} \Sigma_{t_{j}}^{2} \int_{t_{j}}^{t_{j+1}}\left(W_{t}-W_{t_{j}}\right) \int_{t_{j}}^{t} \int_{t_{j}}^{u} \hat{\Gamma}_{s} \mathrm{~d} W_{s} \mathrm{~d} W_{u} \mathrm{~d} t\right|^{2}\right] \\
& \leq C n^{3} \sum_{j} E\left[\Gamma_{t_{j}}^{2} \Sigma_{t_{j}}^{4}\left|\int_{t_{j}}^{t_{j+1}}\left(W_{t}-W_{t_{j}}\right) \int_{t_{j}}^{t} \int_{t_{j}}^{u} \hat{\Gamma}_{s} \mathrm{~d} W_{s} \mathrm{~d} W_{u} \mathrm{~d} t\right|^{2}\right] \\
& \leq C n^{2} \sum_{j} \int_{t_{j}}^{t_{j+1}} E\left[\Gamma_{t_{j}}^{2} \Sigma_{t_{j}}^{4}\left(W_{t}-W_{t_{j}}\right)^{2}\left(\int_{t_{j}}^{t} \int_{t_{j}}^{u} \hat{\Gamma}_{s} \mathrm{~d} W_{s} \mathrm{~d} W_{u}\right)^{2}\right] \mathrm{d} t \\
& \leq C n^{2} E\left[\int_{0}^{T}\left(\Gamma \Sigma^{2}\right)_{[n t] / n}^{8} \mathrm{~d} t\right]^{1 / 4} E\left[\int_{0}^{T}\left|W_{t}-W_{[n t] / n}\right|^{8} \mathrm{~d} t\right]^{1 / 4} \\
& \left.\leq\left. C n E\left[\left.\left.\int_{0}^{T}\left|\int_{[n t] / n}^{t}\right| \int_{[n t] / t}^{u} \hat{\Gamma}_{s} \mathrm{~d} W_{s}\right|^{2} \mathrm{~d} u\right|_{[n t] / n} ^{t} \mathrm{~d} t\right]_{[n t] / t}^{u} \hat{\Gamma}_{s} \mathrm{~d} W_{s} \mathrm{~d} W_{u}\right|^{4} \mathrm{~d} t\right]^{1 / 2} \\
& \leq C n^{1 / 2} E\left[\int_{0}^{T} \int_{[n t] / n}^{t}\left|\int_{[n t] / t}^{u} \hat{\Gamma}_{s} \mathrm{~d} W_{s}\right|^{4} \mathrm{~d} u \mathrm{~d} t\right]^{1 / 2} \\
& \leq C n^{1 / 2} E\left[\int_{0}^{T} \int_{[n t] / n}^{t}\left|\int_{[n t] / t}^{u} \hat{\Gamma}_{s}^{2} \mathrm{~d} s\right|^{2} \mathrm{~d} u \mathrm{~d} t\right]^{1 / 2} \leq \frac{C}{n}\|\hat{\Gamma}\|_{4}^{2} \rightarrow 0 .
\end{aligned}
$$

Step 4). Next, we observe that

$$
n^{3 / 2} \sum_{j} \int_{t_{j}}^{t_{j+1}}\left(\int_{t_{j}}^{t}\left(\Gamma_{u}-\Gamma_{t_{j}}\right) \mathrm{d} W_{u}\right)^{2} \Sigma_{t}^{2} \mathrm{~d} t
$$

is negligible. This simply follows from

$$
E\left[\left|\Gamma_{u}-\Gamma_{t_{j}}\right|^{4}\right] \leq C\left|u-t_{j}\right|^{2}
$$

and so, we omit the detail.

Step 5). It remains to show that the part involved with $\Xi$;

$$
n^{3 / 2} \sum_{j} \int_{t_{j}}^{t_{j+1}}\left(\int_{t_{j}}^{t} \Xi_{u} \mathrm{~d} u\right)^{2} \Sigma_{t}^{2} \mathrm{~d} t+2 n^{3 / 2} \sum_{j} \int_{t_{j}}^{t_{j+1}} \int_{t_{j}}^{t} \Xi_{u} \mathrm{~d} u \int_{t_{j}}^{t} \Gamma_{u} \mathrm{~d} W_{u} \Sigma_{t}^{2} \mathrm{~d} t
$$

is negligible. The first term is easy to treat. For the second term, we first observe that it can be approximated by

$$
2 n^{3 / 2} \sum_{j} \int_{t_{j}}^{t_{j+1}} \Xi_{t_{j}}\left(t-t_{j}\right) \Gamma_{t_{j}}\left(W_{t}-W_{t_{j}}\right) \Sigma_{t_{j}}^{2} \mathrm{~d} t
$$


by a similar argument as before. Then, using that

$$
E_{t_{j}}\left[\int_{t_{j}}^{t_{j+1}} \Xi_{t_{j}}\left(t-t_{j}\right) \Gamma_{t_{j}}\left(W_{t}-W_{t_{j}}\right) \Sigma_{t_{j}}^{2} \mathrm{~d} t\right]=0
$$

we have

$$
\begin{aligned}
& 4 n^{3} E\left[\left|\sum_{j} \int_{t_{j}}^{t_{j+1}} \Xi_{t_{j}}\left(t-t_{j}\right) \Gamma_{t_{j}}\left(W_{t}-W_{t_{j}}\right) \Sigma_{t_{j}}^{2} \mathrm{~d} t\right|^{2}\right] \\
& =4 n^{3} E\left[\sum_{j}\left|\int_{t_{j}}^{t_{j+1}} \Xi_{t_{j}}\left(t-t_{j}\right) \Gamma_{t_{j}}\left(W_{t}-W_{t_{j}}\right) \Sigma_{t_{j}}^{2} \mathrm{~d} t\right|^{2}\right] \\
& \leq 4 n^{2} \sum_{j} E\left[\Xi_{t_{j}}^{2} \Gamma_{t_{j}}^{2} \Sigma_{t_{j}}^{4} \int_{t_{j}}^{t_{j+1}}\left(t-t_{j}\right)^{2}\left(W_{t}-W_{t_{j}}\right)^{2} \mathrm{~d} t\right] \\
& =n^{2} \sum_{j} E\left[\Xi_{t_{j}}^{2} \Gamma_{t_{j}}^{2} \Sigma_{t_{j}}^{4}\right]\left(t_{j+1}-t_{j}\right)^{4} \rightarrow 0
\end{aligned}
$$

since $t_{j}=t_{j}^{n} \wedge T$.

\subsection{Limit results for $\left(V^{n}, Z^{n}\right)$}

Lemma 5.4 Consider $p>1$ and $n \geq 1$ and assume that (H1) holds. Then $V^{n} \in \mathbb{L}_{-}^{1, p}$, $D^{-} V^{n} \in \mathbb{L}^{1, p}$ and, for any $\alpha>0$ and $\beta<1$ there exists a positive constant $C$ such that

$$
\begin{gathered}
\left\|V^{n}\right\|_{1, p}<C n^{\alpha}, \\
\left\|D^{-} V^{n}\right\|_{1, p}<C n^{\alpha}
\end{gathered}
$$

and

$$
\left\|D_{r} V_{t}^{n}-D_{r} V_{0}^{n}\right\|_{p}<C n^{\alpha}(t-r)^{\beta} .
$$

Proof: We know that

$$
V_{t}^{n}=n \int_{t}^{T}\left(X_{s}-X_{s}^{n}\right)^{2} \Sigma_{s}^{2} \mathrm{~d} s
$$

Remark 3.2 gives us that $\Sigma^{2} \in \mathbb{L}^{1, q}$ for every $q>1$. On the other hand, Proposition 2.1. Burkhölder-Davis-Gundy inequality and Hölder's inequality give us that, for every $q>1$ and $\gamma>0$, there exist a constant $C>0$ such that

$$
\left\|X-X^{n}\right\|_{1, q} \leq C n^{\gamma-1 / 2} \text {. }
$$

Now (7) results follows from a direct application of Hölder's inequaliy. A similar argument gives us (8). Finally, we can write

$$
D_{r} V_{t}^{n}-D_{r} V_{0}^{n}=-n \int_{r}^{t} D_{r}\left[\left(X_{s}-X_{s}^{n}\right)^{2} \Sigma_{s}^{2}\right] \mathrm{d} s,
$$

which, jointly with Burkhölder-Davis-Gundy and Hölder inequalities, gives us (9). Now the proof is complete. 
Lemma 5.5 Assume that (H1) holds. Then, for every $n>1$, there exists an adapted and square integrable process $R^{n}$ satisfying that

$$
D^{-} V_{s}^{n}=R_{s}^{n}+n \int_{s}^{T}\left(\int_{\frac{[n \theta]}{n}}^{\theta} D_{s}\left(\Gamma_{r}^{2} \Sigma_{\theta}^{2}\right) d r\right) \mathrm{d} \theta,
$$

where, for any $\delta<1$, there exist two constants $C$ and $p>1$ such that, for any $A \in \mathbb{D}^{2, p}$ and $s \in[0, T]$

$$
\left|E\left(A R_{s}^{n}\right)\right| \leq C n^{-\delta}\|A\|_{2, p}
$$

Proof: Note that

$$
\begin{aligned}
D^{-} V_{s}^{n}= & 2 n \int_{s}^{T}\left(X_{\theta}-X_{\theta}^{n}\right)\left[D_{s}\left(X_{\theta}-X_{\theta}^{n}\right)\right] \Sigma_{\theta}^{2} \mathrm{~d} \theta \\
& +n \int_{s}^{T}\left(X_{\theta}-X_{\theta}^{n}\right)^{2} D_{s} \Sigma_{\theta}^{2} \mathrm{~d} \theta
\end{aligned}
$$

Now, as

$$
Y_{\theta}^{n}:=X_{\theta}-X_{\theta}^{n}=\int_{\frac{[n \theta]}{n}}^{\theta} \Xi_{\tau} \mathrm{d} \tau+\int_{\frac{[n \theta]}{n}}^{\theta} \Gamma_{\tau} \mathrm{d} W_{\tau}
$$

and

$$
U_{\theta}^{n}:=D_{s}\left(X_{\theta}-X_{\theta}^{n}\right)=\mathbf{1}_{\left[\frac{[n \theta]}{n}, \theta\right]}(s) \Gamma_{s}+\int_{\frac{[n \theta \theta]}{n}}^{\theta} D_{s} \Xi_{\tau} \mathrm{d} \tau+\int_{\frac{[n \theta]}{n}}^{\theta} D_{s} \Gamma_{\tau} \mathrm{d} W_{\tau}
$$

Itô's formula gives us that

$$
\begin{aligned}
& D^{-} V_{s}^{n}=2 n \int_{s}^{T} Y_{\theta}^{n} U_{\theta}^{n} \Sigma_{\theta}^{2} \mathrm{~d} \theta+n \int_{s}^{T}\left(Y_{\theta}^{n}\right)^{2} D_{s} \Sigma_{\theta}^{2} \mathrm{~d} \theta \\
& =2 n \int_{s}^{T}\left(X_{\theta}-X_{\theta}^{n}\right) \mathbf{1}_{\left[\frac{[n \theta]}{n}, \theta\right]}(s) \Gamma_{s} \Sigma_{\theta}^{2} \mathrm{~d} \theta \\
& +2 n \int_{s}^{T}\left(\int_{\frac{[n \theta]}{n}}^{\theta} \Xi_{\tau} \hat{U}_{\tau}^{n} \mathrm{~d} \tau\right) \Sigma_{\theta}^{2} \mathrm{~d} \theta+2 n \int_{s}^{T}\left(\int_{\frac{[n \theta]}{n}}^{\theta} \Gamma_{\tau} \hat{U}_{\tau}^{n} \mathrm{~d} W_{\tau}\right) \Sigma_{\theta}^{2} \mathrm{~d} \theta \\
& +2 n \int_{s}^{T}\left(\int_{\frac{[n \theta]}{n}}^{\theta} D_{s} \Xi_{\tau} Y_{\tau}^{n} \mathrm{~d} \tau\right) \Sigma_{\theta}^{2} \mathrm{~d} \theta+2 n \int_{s}^{T}\left(\int_{\frac{[n \theta]}{n}}^{\theta} D_{s} \Gamma_{\tau} Y_{\tau}^{n} \mathrm{~d} W_{\tau}\right) \Sigma_{\theta}^{2} \mathrm{~d} \theta \\
& +2 n \int_{s}^{T}\left(\int_{\frac{[n \theta]}{n}}^{\theta} \Xi_{\tau} Y_{\tau}^{n} \mathrm{~d} \tau\right) D_{s} \Sigma_{\theta}^{2} \mathrm{~d} \theta+2 n \int_{s}^{T}\left(\int_{\frac{[n \theta]}{n}}^{\theta} \Gamma_{\tau} Y_{\tau}^{n} \mathrm{~d} W_{\tau}\right) D_{s} \Sigma_{\theta}^{2} \mathrm{~d} \theta \\
& +2 n \int_{s}^{T}\left(\int_{\frac{[n \theta]}{n}}^{\theta} \Gamma_{\tau} D_{s} \Gamma_{\tau} \mathrm{d} \tau\right) \Sigma_{\theta}^{2} \mathrm{~d} \theta+n \int_{s}^{T}\left(\int_{\frac{[n \theta \theta}{n}}^{\theta} \Gamma_{\tau}^{2} \mathrm{~d} \tau\right) D_{s} \Sigma_{\theta}^{2} \mathrm{~d} \theta \\
& =: R_{s}^{n}+2 n \int_{s}^{T}\left(\int_{\frac{[n \theta]}{n}}^{\theta} \Gamma_{\tau} D_{s} \Gamma_{\tau} \mathrm{d} \tau\right) \Sigma_{\theta}^{2} \mathrm{~d} \theta+n \int_{s}^{T}\left(\int_{\frac{[n \theta]]}{n}}^{\theta} \Gamma_{\tau}^{2} \mathrm{~d} \tau\right) D_{s} \Sigma_{\theta}^{2} \mathrm{~d} \theta \\
& =: R_{s}^{n}+n \int_{s}^{T}\left(\int_{\frac{[n \theta]]}{n}}^{\theta} D_{s}\left(\Gamma_{\tau}^{2} \Sigma_{\theta}^{2}\right) \mathrm{d} \tau\right) \mathrm{d} \theta,
\end{aligned}
$$


where $\hat{U}_{\tau}^{n}=U_{\tau}^{n}-\mathbf{1}_{\left[\frac{[n \theta]}{n}, \theta\right]}(s) \Gamma_{s}$. Now, using again the duality relationship and Hölder's inequality it is easy to see that

$$
\left|E\left(A R_{s}^{n}\right)\right| \leq C n^{-1+\frac{2}{q}}\|A\|_{2, p}
$$

for any $p>q>1$. Now, taking $q>\frac{2}{1-\delta}$ the proof is complete.

Lemma 5.6

$$
\lim _{n \rightarrow \infty} n \int_{0}^{T} \int_{[n u] / n}^{u} E\left[q_{1,1}\left(V_{0}^{n}, 0\right)\left(D^{-} V_{s}^{n}-D^{-} V_{s}\right) \Theta_{u} \Gamma_{s}\right] \mathrm{d} s \mathrm{~d} u=0 .
$$

Proof: Lemma5.5gives us that

$$
\begin{aligned}
& n \int_{0}^{T} \int_{[n u] / n}^{u} E\left[q_{1,1}\left(V_{0}^{n}, 0\right)\left(D^{-} V_{s}^{n}-D^{-} V_{s}\right) \Theta_{u} \Gamma_{s}\right] \mathrm{d} s \mathrm{~d} u \\
& =n \int_{0}^{T} \int_{[n u] / n}^{u} E\left[q_{1,1}\left(V_{0}^{n}, 0\right) R_{s}^{n} \Theta_{u} \Gamma_{s}\right] \mathrm{d} s \mathrm{~d} u \\
& +n^{2} \int_{0}^{T} \int_{[n u] / n}^{u} E\left[q_{1,1}\left(V_{0}^{n}, 0\right)\left[\left(\int_{s}^{T} \int_{\frac{[n \theta]}{n}}^{\theta} D_{s}\left(\Gamma_{\tau}^{2} \Sigma_{\theta}^{2}\right) \mathrm{d} \tau \mathrm{d} \theta\right)-\frac{1}{n} D^{-} V_{s}\right] \Theta_{u} \Gamma_{s}\right] \mathrm{d} s \mathrm{~d} u .
\end{aligned}
$$

(12) gives us that the first term in the right-hand side of the above equatlity tends to zero. On the other hand, for any $s<\theta$, the process $D_{s}\left(\Gamma^{2} \Sigma_{\theta}^{2}\right)$ is continuous in $L^{p}(\Omega)$. Then, a direct application of Hölder's inequality gives us that the second term tends to zero. Now the proof is complete.

\section{Lemma 5.7}

$$
n \int_{0}^{T} E\left[q_{1,1}\left(V_{0}^{n}, 0\right)\left(D^{-} V_{t}^{n}-D^{-} V_{t}\right)\left(X_{t}-X_{t}^{n}\right) \Sigma_{t}\right] \mathrm{d} t \rightarrow \frac{1}{3} E\left[q_{1,1}\left(V_{0}, 0\right) \int_{0}^{T} \Sigma_{t}^{3} \Gamma_{t}^{3} \mathrm{~d} t\right] .
$$

Proof: We can make use of of the computations in the proof of Lemma 5.5 to see that the leading terms should be

$$
\begin{aligned}
& 2 n^{2} \int_{0}^{T}\left(\int_{\frac{[n s]}{n}}^{s} E\left[q_{1,1}\left(V_{0}^{n}, 0\right)\left(X_{s}-X_{s}^{n}\right) \Gamma_{t} \Sigma_{s}^{2}\left(X_{t}-X_{t}^{n}\right) \Sigma_{t}\right] \mathrm{d} t\right) \mathrm{d} s \\
& +n^{2} \int_{0}^{T} E\left[q_{1,1}\left(V_{0}^{n}, 0\right)\left(\int_{t}^{T} \int_{\frac{[n s]}{n}}^{s} D_{t}\left(\Gamma_{\tau}^{2} \Sigma_{s}^{2}\right) \mathrm{d} \tau \mathrm{d} s-\frac{1}{n} D^{-} V_{t}\right)\left(X_{t}-X_{t}^{n}\right) \Sigma_{t}\right] \mathrm{d} t .
\end{aligned}
$$

By the duality relationship, the second term tends to zero. For the first one, we have that its limit is

$$
\begin{aligned}
& 2 \lim _{n \rightarrow \infty} n^{2} E \sum_{i=1}^{n} \int_{t_{i}}^{t_{i+1}} \int_{\frac{[n s]}{n}}^{s} E\left[q_{1,1}\left(V_{0}^{n}, 0\right)\left(X_{s}-X_{s}^{n}\right) \Gamma_{s} \Sigma_{s}^{2}\left(X_{t}-X_{t}^{n}\right) \Sigma_{t}\right] \mathrm{d} t \mathrm{~d} s \\
& =2 \lim _{n \rightarrow \infty} n^{2} E \sum_{i=1}^{n} \int_{t_{i}}^{t_{i+1}} \int_{\frac{[n s]}{n}}^{s} E\left[q_{1,1}\left(V_{0}^{n}, 0\right) \Gamma_{s} \Sigma_{s}^{2}\left(\int_{t_{i}}^{t} \Gamma_{r}^{2} \mathrm{~d} r\right) \Sigma_{t}\right] \mathrm{d} t \mathrm{~d} s \\
& =\frac{1}{3} \lim _{n \rightarrow \infty} E\left[q_{1,1}\left(V_{0}^{n}, 0\right) \int_{0}^{T} \Sigma_{t}^{3} \Gamma_{t}^{3} \mathrm{~d} t\right],
\end{aligned}
$$


and this allows us to complete the proof.

The proofs of Lemmas 6.2 and 6.3 will be based on the following technical result.

Lemma 5.8 Suppose that (H1) holds. Consider a real function $f=q_{i, j}$, for some $i, j \geq 0$. Let $p>1$. Then, for any $\alpha>1 / p$, there exists $C>0$ such that for any $A \in \mathbb{D}^{2, p}$,

$$
E\left[\left(f\left(V_{t}^{n}, Z_{t}^{n}\right)-f\left(V_{0}^{n}, 0\right)\right) A\right] \leq C n^{\alpha-\frac{1}{2}}\|A\|_{2, p} .
$$

Proof: Using that $f=q_{i, j}$ solves the heat equation, a direct application of the anticipating Itô's formula gives us that

$$
\begin{aligned}
& A f\left(V_{t}^{n}, Z_{t}^{n}\right)-A f\left(V_{0}^{n}, 0\right) \\
& =\sqrt{n} \int_{0}^{t} A \partial_{y} f\left(V_{s}^{n}, Z_{s}^{n}\right)\left(X_{s}-X_{s}^{n}\right) \mathrm{d} Y_{s} \\
& +\sqrt{n} \int_{0}^{t} A \partial_{x y}^{2} f\left(V_{s}^{n}, Z_{s}^{n}\right) D^{-} V_{s}^{n}\left(X_{s}-X_{s}^{n}\right) \Sigma_{s} \mathrm{~d} s \\
& +\sqrt{n} \int_{0}^{t} \partial_{y} f\left(V_{s}^{n}, Z_{s}^{n}\right) D_{s} A\left(X_{s}-X_{s}^{n}\right) \Sigma_{s} \mathrm{~d} s .
\end{aligned}
$$

Then, taking conditional expectations we get

$$
\begin{aligned}
& E\left[\left(f\left(V_{t}^{n}, Z_{t}^{n}\right)-f\left(V_{0}^{n}, 0\right)\right) A\right] \\
& =\sqrt{n} E\left[\int_{0}^{t} A \partial_{y} f\left(V_{s}^{n}, Z_{s}^{n}\right)\left(X_{s}-X_{s}^{n}\right) \Theta_{s} \mathrm{~d} s\right. \\
& \left.+\int_{0}^{t} A \partial_{x y}^{2} f\left(V_{s}^{n}, Z_{s}^{n}\right) D^{-} V_{s}^{n}\left(X_{s}-X_{s}^{n}\right) \Sigma_{s} \mathrm{~d} s+\int_{0}^{t} \partial_{y} f\left(V_{s}^{n}, Z_{s}^{n}\right) D_{s} A\left(X_{s}-X_{s}^{n}\right) \sum_{s} \mathrm{~d} s\right] \\
& =: \sqrt{n} \int_{0}^{t}\left(X_{s}-X_{s}^{n}\right) J_{s} \mathrm{~d} s,
\end{aligned}
$$

where

$$
J_{s}:=A \partial_{y} f\left(V_{s}^{n}, Z_{s}^{n}\right) \Theta_{s}+A \partial_{x y}^{2} f\left(V_{s}^{n}, Z_{s}^{n}\right) D^{-} V_{s}^{n} \Sigma_{s}+\partial_{y} f\left(V_{s}^{n}, Z_{s}^{n}\right) D_{s} A \Sigma_{s} .
$$

Now, the duality relationship between the Skorohod integral and the Malliavin derivative operator give us that

$$
\begin{aligned}
& E\left[\left(f\left(V_{t}^{n}, Z_{t}^{n}\right)-f\left(V_{0}^{n}, 0\right)\right) A\right] \\
& =\sqrt{n} E\left[\int_{0}^{t} \int_{\frac{[n t]}{n}}^{t} J_{s} \Xi_{r} \mathrm{~d} s \mathrm{~d} r+\int_{0}^{t} \int_{\frac{[n t]}{n}}^{t}\left(D_{r} J_{s}\right) \Gamma_{r} \mathrm{~d} s \mathrm{~d} r\right] \\
& \leq n^{-\frac{1}{2}+\alpha} E\left[\int_{0}^{t} \int_{\frac{[n t}{n}}^{t}\left|J_{s} \Xi_{r}\right|^{\frac{1}{\alpha}} \mathrm{d} s \mathrm{~d} r+\int_{0}^{t} \int_{\frac{[n t}{n}}^{t}\left|\left(D_{r} J_{s}\right) \Gamma_{r}\right|^{\frac{1}{\alpha}} \mathrm{d} s \mathrm{~d} r\right]^{\alpha}
\end{aligned}
$$

for any $\alpha>1 / p$. Then, the result follows as a direct consequence of (H1), Lemma 5.4 and Hölder's inequality. 


\section{The leading terms}

Now we are in a position to prove the limit lemmas.

\section{Lemma 6.1}

$$
\sqrt{n}\left\{E\left[q\left(V_{0}^{n}, 0\right)\right]-E\left[q\left(V_{0}, 0\right)\right]\right\} \rightarrow 0
$$

as $n \rightarrow \infty$.

Proof: By Taylor's formula,

$$
\sqrt{n}\left\{E\left[q\left(V_{0}^{n}, 0\right)\right]-E\left[q\left(V_{0}, 0\right)\right]\right\}=E\left[\sqrt{n}\left(V_{0}^{n}-V_{0}\right) \int_{0}^{1} q_{1,0}\left(V_{0}+\left(V_{0}^{n}-V_{0}\right) s, 0\right) \mathrm{d} s\right] .
$$

Since $q_{1,0}$ is bounded, the result follows from Proposition 5.1.

\section{Lemma 6.2}

$$
\begin{aligned}
& n \int_{0}^{T} E\left[q_{0,1}\left(V_{t}^{n}, Z_{t}^{n}\right)\left(X_{t}-X_{t}^{n}\right) \Theta_{t}\right] \mathrm{d} t \\
& \rightarrow \frac{1}{2} \int_{0}^{T} E\left[q_{0,1}\left(V_{0}, 0\right)\left(\Xi_{t} \Theta_{t}+D^{+} \Theta_{t} \Gamma_{t}\right)\right] \mathrm{d} t+\frac{1}{2} \int_{0}^{T} E\left[q_{1,1}\left(V_{0}, 0\right) D^{-} V_{t} \Theta_{t} \Gamma_{t}\right] \mathrm{d} t
\end{aligned}
$$

as $n \rightarrow \infty$.

Proof: We can write

$$
\begin{aligned}
& n \int_{0}^{T} E\left[q_{0,1}\left(V_{t}^{n}, Z_{t}^{n}\right)\left(X_{t}-X_{t}^{n}\right) \Theta_{t}\right] \mathrm{d} t \\
& =n \int_{0}^{T} E\left[q_{0,1}\left(V_{t}^{n}, Z_{t}^{n}\right)\left(\int_{\frac{[n t]}{n}}^{t} \Xi_{r} \mathrm{~d} r\right) \Theta_{t}\right] \mathrm{d} t+n \int_{0}^{T} E\left[q_{0,1}\left(V_{t}^{n}, Z_{t}^{n}\right)\left(\int_{\frac{[n t]}{n}}^{t} \Gamma_{r} \mathrm{~d} W_{r}\right) \Theta_{t}\right] \mathrm{d} t \\
& =n \int_{0}^{T} E\left[q_{0,1}\left(V_{0}^{n}, 0\right)\left(\int_{\frac{[n t]}{n}}^{t} \Xi_{r} \mathrm{~d} r\right) \Theta_{t}\right] \mathrm{d} t+n \int_{0}^{T} E\left[q_{0,1}\left(V_{0}^{n}, 0\right)\left(\int_{\frac{[n t]}{n}}^{t} \Gamma_{r} \mathrm{~d} W_{r}\right) \Theta_{t}\right] \mathrm{d} t \\
& +n \int_{0}^{T} E\left[\left(q_{0,1}\left(V_{t}^{n}, Z_{t}^{n}\right)-q_{0,1}\left(V_{0}^{n}, 0\right)\right)\left(\int_{\frac{[n t}{n}}^{t} \Xi_{r} \mathrm{~d} r\right) \Theta_{t}\right] \mathrm{d} t \\
& +n \int_{0}^{T} E\left[\left(q_{0,1}\left(V_{t}^{n}, Z_{t}^{n}\right)-q_{0,1}\left(V_{0}^{n}, 0\right)\right)\left(\int_{\frac{[n t}{n}}^{t} \Gamma_{r} \mathrm{~d} W_{r}\right) \Theta_{t}\right] \mathrm{d} t \\
& =: T_{1}+T_{2}+T_{3}+T_{4} .
\end{aligned}
$$

Now,

$$
T_{1} \rightarrow \frac{1}{2} E\left[q_{0,1}\left(V_{0}, 0\right) \int_{0}^{T} \Xi_{t} \Theta_{t} \mathrm{~d} t\right]
$$


since

$$
\begin{aligned}
& E\left|\int_{0}^{T} n \int_{[n u] / n}^{u}\left[q_{0,1}\left(V_{0}^{n}, 0\right) \Xi_{s} \Theta_{u}\right] \mathrm{d} s \mathrm{~d} u-\frac{1}{2} \int_{0}^{T}\left[q_{0,1}\left(V_{0}, 0\right) \Xi_{t} \Theta_{t}\right] \mathrm{d} t\right| \\
& \leq E\left|q_{0,1}\left(V_{0}^{n}, 0\right)\left(\int_{0}^{T} n \int_{[n u] / n}^{u} \Theta_{u} \Xi_{s} \mathrm{~d} s \mathrm{~d} u-\frac{1}{2} \int_{0}^{T} \Xi_{u} \Theta_{u} \mathrm{~d} u\right)\right| \\
& +\frac{1}{2} E\left|\left(q_{0,1}\left(V_{0}^{n}, 0\right)-q_{0,1}\left(V_{0}^{n}, 0\right)\right) \int_{0}^{T} \Xi_{t} \Theta_{t} \mathrm{~d} t\right|
\end{aligned}
$$

which tends to zero due to (H1), (H3), Lemma5.1 and Proposition 5.1.

For the second term, by the duality between the Malliavin derivative and the Skorohod integral,

$$
\begin{aligned}
& T_{2}=n \int_{0}^{T} \int_{[n u] / n}^{u} E\left[D_{s}\left[q_{0,1}\left(V_{0}^{n}, 0\right) \Theta_{u}\right] \Gamma_{s}\right] \mathrm{d} s \mathrm{~d} u \\
& =n \int_{0}^{T} \int_{[n u] / n}^{u} E\left[\left(q_{1,1}\left(V_{0}^{n}, 0\right) D^{-} V_{s}^{n} \Theta_{u}+q_{0,1}\left(V_{0}^{n}, 0\right) D_{s} \Theta_{u}\right) \Gamma_{s}\right] \mathrm{d} s \mathrm{~d} u \\
& \left.=n \int_{0}^{T} \int_{[n u] / n}^{u} E\left[\left(q_{1,1}\left(V_{0}^{n}, 0\right) D^{-} V_{s} \Theta_{u}\right) \Gamma_{s}\right]\right] \mathrm{d} s \mathrm{~d} u \\
& +n \int_{0}^{T} \int_{[n u] / n}^{u} E\left[\left(q_{0,1}\left(V_{0}^{n}, 0\right) D_{s}^{+} \Theta_{u}\right) \Gamma_{s}\right] \mathrm{d} s \mathrm{~d} u \\
& +n \int_{0}^{T} \int_{[n u] / n}^{u} E\left[\left(q_{1,1}\left(V_{0}^{n}, 0\right)\left(D^{-} V_{s}^{n}-D^{-} V_{s}\right) \Theta_{u}\right) \Gamma_{s}\right] \mathrm{d} s \mathrm{~d} u \\
& +n \int_{0}^{T} \int_{[n u] / n}^{u} E\left[q_{0,1}\left(V_{0}^{n}, 0\right)\left(D_{s}^{+} \Theta_{u}-D_{s} \Theta_{u}\right) \Gamma_{s}\right] \mathrm{d} s \mathrm{~d} u
\end{aligned}
$$

Notice that (H1), (H2) and Lemma5.6imply that the last two terms in the above equality tend to zero. Then, similar arguments as for $T_{1}$ give us that

$$
T_{2} \rightarrow \frac{1}{2} \int_{0}^{T} E\left[q_{1,1}\left(V_{0}, 0\right) D^{-} V_{t} \Theta_{t} \Gamma_{t}\right] \mathrm{d} t+\frac{1}{2} \int_{0}^{T} E\left[q_{0,1}\left(V_{0}, 0\right) D^{+} \Theta_{t} \Gamma_{t}\right] \mathrm{d} t
$$

Let us study $T_{3}$. We have that

$$
\begin{aligned}
& T_{3}=n \int_{0}^{T} E\left[\left(q_{0,1}\left(V_{t}^{n}, Z_{t}^{n}\right)-q_{0,1}\left(V_{0}^{n}, 0\right)\right)\left(\int_{\frac{[n t]}{n}}^{t} \Xi_{r} \mathrm{~d} r\right) \Theta_{t}\right] \mathrm{d} t \\
& =n \int_{0}^{T} E \int_{\frac{[n t]}{n}}^{t}\left(q_{0,1}\left(V_{t}^{n}, Z_{t}^{n}\right)-q_{0,1}\left(V_{0}^{n}, 0\right)\right) \Xi_{r} \Theta_{t} \mathrm{~d} r \mathrm{~d} t,
\end{aligned}
$$


which tends to zero due to Lemma5.8 For the last term,

$$
\begin{aligned}
& T_{4}=n \int_{0}^{T} E\left[\left(q_{0,1}\left(V_{t}^{n}, Z_{t}^{n}\right)-q_{0,1}\left(V_{0}^{n}, 0\right)\right)\left(\int_{\frac{[n t]}{n}}^{t} \Gamma_{r} \mathrm{~d} W_{r}\right) \Theta_{t}\right] \mathrm{d} t \\
& =n \int_{0}^{T} E\left[\int_{\frac{[n t]}{n}}^{t} D_{r}\left[\Theta_{t}\left(q_{0,1}\left(V_{t}^{n}, Z_{t}^{n}\right)-q_{0,1}\left(V_{0}^{n}, 0\right)\right)\right] \Gamma_{r} \mathrm{~d} r\right] \mathrm{d} t \\
& =n \int_{0}^{T} E\left[\int_{\frac{[n t]}{n}}^{t}\left(D_{r} \Theta_{t}\right)\left(q_{0,1}\left(V_{t}^{n}, Z_{t}^{n}\right)-q_{0,1}\left(V_{0}^{n}, 0\right)\right) \Gamma_{r} \mathrm{~d} r\right] \mathrm{d} t \\
& +n \int_{0}^{T} E\left[\int_{\frac{[n t]}{n}}^{t}\left[\Theta_{t} D_{r}\left(q_{0,1}\left(V_{t}^{n}, Z_{t}^{n}\right)-q_{0,1}\left(V_{0}^{n}, 0\right)\right)\right] \Gamma_{r} \mathrm{~d} r\right] \mathrm{d} t \\
& =: T_{4}^{1}+T_{4}^{2} .
\end{aligned}
$$

Using again (H1) and Lemma 5.8, we can easily check that $T_{4}^{1} \rightarrow 0$. Now,

$$
\begin{aligned}
& T_{4}^{2}=n \int_{0}^{T} E\left[\int_{\frac{[n t}{n}}^{t} \Theta_{t}\left(q_{1,1}\left(V_{t}^{n}, Z_{t}^{n}\right) D_{r} V_{t}^{n}-q_{1,1}\left(V_{0}^{n}, 0\right) D_{r} V_{0}^{n}\right) \Gamma_{r} \mathrm{~d} r\right] \mathrm{d} t \\
& +n \int_{0}^{T} E\left[\int_{\frac{[n t]}{n}}^{t} \Theta_{t}\left(q_{0,2}\left(V_{t}^{n}, Z_{t}^{n}\right) D_{r} Z_{t}^{n}\right) \Gamma_{r} \mathrm{~d} r\right] \mathrm{d} t \\
& =n \int_{0}^{T} E\left[\int_{\frac{[n t]}{n}}^{t} \Theta_{t}\left(q_{1,1}\left(V_{t}^{n}, Z_{t}^{n}\right)-q_{1,1}\left(V_{0}^{n}, 0\right)\right) D_{r} V_{t}^{n} \Gamma_{r} \mathrm{~d} r\right] \mathrm{d} t \\
& +n \int_{0}^{T} E\left[\int_{\frac{[n t]}{n}}^{t} \Theta_{t} q_{1,1}\left(V_{0}^{n}, 0\right)\left(D_{r} V_{t}^{n}-D_{r} V_{0}^{n}\right) \Gamma_{r} \mathrm{~d} r\right] \mathrm{d} t \\
& +n \int_{0}^{T} E\left[\int_{\frac{[n t]}{n}}^{t} \Theta_{t}\left(q_{0,2}\left(V_{t}^{n}, Z_{t}^{n}\right) D_{r} Z_{t}^{n}\right) \Gamma_{r} \mathrm{~d} r\right] \mathrm{d} t \\
& =: T_{4}^{2,1}+T_{4}^{2,2}+T_{4}^{2,3} .
\end{aligned}
$$

The same arguments as before, together with Lemma 5.4 gives us that $T_{4}^{2,1}+$ $T_{4}^{2,2} \rightarrow 0$. On the other hand,

$$
\begin{aligned}
& D_{r} Z_{t}^{n} \\
= & \sqrt{n} D_{r}\left[\int_{0}^{t}\left(X_{s}-X_{s}^{n}\right) \Theta_{s} \mathrm{~d} s+\int_{0}^{t}\left(X_{s}-X_{s}^{n}\right) \Sigma_{s} \mathrm{~d} W_{s}\right] \\
= & \sqrt{n}\left[\int_{r}^{t} D_{r}\left(X_{s}-X_{s}^{n}\right) \Theta_{s} \mathrm{~d} s+\int_{r}^{t}\left(X_{s}-X_{s}^{n}\right) D_{r} \Theta_{s} \mathrm{~d} s\right. \\
+ & \left.\left(X_{r}-X_{r}^{n}\right) \Sigma_{r}+\int_{r}^{t} D_{r}\left(X_{s}-X_{s}^{n}\right) \Sigma_{s} \mathrm{~d} W_{s}+\int_{r}^{t}\left(X_{s}-X_{s}^{n}\right) D_{r} \Sigma_{s} \mathrm{~d} W_{s}\right] \\
=: & \left(X_{r}-X_{r}^{n}\right) \Sigma_{r}+H_{r, t} .
\end{aligned}
$$


Then

$$
\begin{aligned}
& T_{4}^{2,3}=n \int_{0}^{T} E\left[\int_{\frac{[n t]}{n}}^{t} \Theta_{t}\left(q_{0,2}\left(V_{t}^{n}, Z_{t}^{n}\right)\left(X_{r}-X_{r}^{n}\right) \Sigma_{r}\right) \Gamma_{r} \mathrm{~d} r\right] \mathrm{d} t \\
& +n \int_{0}^{T} E\left[\int_{\frac{[n t}{n}}^{t} \Theta_{t}\left(q_{0,2}\left(V_{t}^{n}, Z_{t}^{n}\right) H_{r, t} \Sigma_{r}\right) \Gamma_{r} \mathrm{~d} r\right] \mathrm{d} t .
\end{aligned}
$$

Using again the duality relationship we deduce that the first term in the righthand side of (20) tends to zero. Moreover, BulkhölderDavis-Gundy and Hölder inequalities give us that for any $p>1, \gamma<1 / 2,\left\|H_{r, t}\right\|_{p} \leq L_{r}(t-r)^{\gamma}$, for some process $L \in L^{p}$. This allows us to complete the proof.

\section{Lemma 6.3}

$$
\begin{aligned}
& n \int_{0}^{T} E\left[q_{1,1}\left(V_{t}^{n}, Z_{t}^{n}\right) D^{-} V_{t}^{n}\left(X_{t}-X_{t}^{n}\right) \Sigma_{t}\right] \mathrm{d} t \\
& \rightarrow \frac{1}{2} \int_{0}^{T} E\left[q_{1,1}\left(V_{0}, 0\right)\left(D^{-} V_{t} \Xi_{t} \Sigma_{t}+\left(D^{-}\right)^{2} V_{t} \Sigma_{t} \Gamma_{t}+D_{t}^{-} V_{t} D^{+} \Sigma_{t} \Gamma_{t}\right)\right] \mathrm{d} t \\
& \quad+\frac{1}{2} \int_{0}^{T} E\left[q_{2,1}\left(V_{0}, 0\right)\left|D^{-} V_{t}\right|^{2} \Gamma_{t} \Sigma_{t}\right] \mathrm{d} t+\frac{1}{3} \int_{0}^{T} E\left[q_{1,1}\left(V_{0}, 0\right) \Sigma_{t}^{3} \Gamma_{t}^{3}\right] \mathrm{d} t
\end{aligned}
$$

as $n \rightarrow \infty$.

Proof: We can write

$$
\begin{aligned}
& n \int_{0}^{T} E\left[q_{1,1}\left(V_{t}^{n}, Z_{t}^{n}\right) D^{-} V_{t}^{n}\left(X_{t}-X_{t}^{n}\right) \Sigma_{t}\right] \mathrm{d} t \\
& =n \int_{0}^{T} E\left[q_{1,1}\left(V_{t}^{n}, Z_{t}^{n}\right) D^{-} V_{t}\left(X_{t}-X_{t}^{n}\right) \Sigma_{t}\right] \mathrm{d} t \\
& +n \int_{0}^{T} E\left[q_{1,1}\left(V_{t}^{n}, Z_{t}^{n}\right)\left(D^{-} V_{t}^{n}-D^{-} V_{t}\right)\left(X_{t}-X_{t}^{n}\right) \Sigma_{t}\right] \mathrm{d} t \\
& =: T_{1}+T_{2} .
\end{aligned}
$$

Notice that $T_{1}$ is similar to the term studied in Lemma 6.2 replacing $q_{0,1}$ by $q_{1,1}$ and $\Theta_{t}$ by $D^{-} V_{t} \Sigma_{t}$. Then, the same arguments as in the proof of the previous result give us that

$$
\begin{aligned}
T_{1} \rightarrow & \frac{1}{2} \int_{0}^{T} E\left[q_{1,1}\left(V_{0}, 0\right)\left(\Xi_{t}\left(D^{-} V_{t} \Sigma_{t}\right)+D^{-}\left(D^{-} V_{t} \Sigma_{t}\right) \Gamma_{t}\right)\right] \mathrm{d} t \\
& +\frac{1}{2} \int_{0}^{T} E\left[q_{2,1}\left(V_{0}, 0\right) D^{-} V_{t}\left(D^{-} V_{t} \Sigma_{t}\right) \Gamma_{t}\right] \mathrm{d} t .
\end{aligned}
$$


This coincides with the first three terms in (21). For the second term

$$
\begin{aligned}
T_{2} \quad & =: n \int_{0}^{T} E\left[q_{1,1}\left(V_{0}^{n}, 0\right)\left(D^{-} V_{t}^{n}-D^{-} V_{t}\right)\left(X_{t}-X_{t}^{n}\right) \Sigma_{t}\right] \mathrm{d} t \\
& +n \int_{0}^{T} E\left[\left(q_{1,1}\left(V_{t}^{n}, Z_{t}^{n}\right)\right)-q_{1,1}\left(V_{0}^{n}, 0\right)\left(D^{-} V_{t}^{n}-D^{-} V_{t}\right)\left(X_{t}-X_{t}^{n}\right) \Sigma_{t}\right] \mathrm{d} t \\
& =: T_{2}^{1}+T_{2}^{2} .
\end{aligned}
$$

Lemma 5.7 gives us that $T_{2}^{1} \rightarrow \frac{1}{3} E\left[q_{1,1}\left(V_{0}, 0\right) \int_{0}^{T} \Sigma_{t}^{3} \Gamma_{t}^{3} d t\right]$. The same arguments as in the proof of this Lemma allow us to prove that $T_{2}^{2}$ tends to zero. Now the proof is complete.

\section{References}

[1] Aït-Sahalia, Y. and Jacod, J. (2014). High-Frequency Financial Econometrics, Prinston University Press.

[2] Fukasawa, M. (2011). Discretization error of stochastic integrals. The Annals of Applied Probability 21, No. 4, 1436-1465.

[3] Jacod, J. and Protter, P.E. (2012). Discretization of Processes, Springer-Verlag Berlin Heidelberg.

[4] Jacod, J. and Shiryaev, A. (2003). Limit Theorems for Stochastic Processes, Springer-Verlag Berlin Heidelberg.

[5] Hall, P. (1992). The Bootstrap and Edgeworth Expansion, Springer-Verlag New York.

[6] Nualart, D. The Malliavin Calculus and Related Topics, Springer, 2006.

[7] Nualart, D. and Pardoux, É. (1988). Stochastic calculus with anticipating integrands. Probab. Theory Relat. Fields 78(4), 535-581.

[8] Podolskij, M. and Yoshida, N. (2016). Edgeworth exansion for functionals of continuous diffusion processes. The Annals of Applied Probability 26, 6, 3415-3455.

[9] Rootzén, H. (1980). Limit distributions for the error in approximations of stochastic integrals. The Annals of Probability 8, No. 2, 241-251.

[10] Yoshida, N. (1997). Malliavin calculus and asymptotic expansion for martingales Probab. Theory Relat. Fields, 109. 301-342.

[11] Yoshida, N. (2001). Malliavin calculus and martingale expansion, Bulletin des Sciences Mathématiques 125, Issues 6-7, 431-456.

[12] Yoshida, N. (2013). Martingale expansion in mixed normal limit. Stochastic Processes and their Applications 123, Issue 3, 887-933. 